\title{
Editorial
}

\section{Multidisciplinary Design Optimization in Engineering}

\author{
Zhuming Bi, ${ }^{1}$ Lihui Wang, ${ }^{2}$ Chong Wu, ${ }^{3}$ Guilin Yang, ${ }^{4}$ and Dan Zhang ${ }^{5}$ \\ ${ }^{1}$ Department of Engineering, Indiana University-Purdue University Fort Wayne, 2101 East Coliseum Boulevard, Fort Wayne, \\ IN 46805-149, USA \\ ${ }^{2}$ Department of Production Engineering, KTH Royal Institute of Technology, Brinellvägen 68, 10044 Stockholm, Sweden \\ ${ }^{3}$ School of Management, Harbin Institute of Technology, Harbin 150001, China \\ ${ }^{4}$ Mechatronics Group, Singapore Institute of Manufacturing Technology, 71 Nanyang Drive, Singapore 638075 \\ ${ }^{5}$ Faculty of Engineering and Applied Science, University of Ontario Institute of Technology, 2000 Simcoe Street, ON, Canada L1H 7K4
}

Correspondence should be addressed to Zhuming Bi; biz@ipfw.edu

Received 20 August 2013; Accepted 20 August 2013

Copyright (C) 2013 Zhuming Bi et al. This is an open access article distributed under the Creative Commons Attribution License, which permits unrestricted use, distribution, and reproduction in any medium, provided the original work is properly cited.

System complexity depends on the number of design parameters in a system as well as the dynamic characteristics of design parameters in the time domain. Complex systems are generally featured as a large number of design parameters, residential dynamic environments, strongly coupled system behaviors, and the needs of multiple design criteria. Modern systems are becoming more and more complex due to numerous factors such as the involvement of multidisciplinary behaviors, increasing functionalities and components, growing amount of information associated with system interactions, and the uncertainties and changes involved in systems.

Multidisciplinary design optimization (MDO) is essential to the design and operation of a complex system. MDO takes into consideration all relevant disciplines simultaneously to find the global optimum which is superior to a solution from a sequence of local optimizations in individual disciplines. MDO has been successfully applied in the designs of many complex systems such as aircrafts, automobiles, shipbuilding, computers, and civic infrastructures. However, a simultaneous consideration of multiple disciplines increases the complexity of design problems, and the products or systems in future tend to be even complicated. Continuing research and development efforts are in demand to advance the theories and methodologies of MDO. This special issue is dedicated to the recent progresses on theories, methodologies, and applications of MDO. The paper solicitation has received numerous responses from the researchers in the field, and, through a rigorous peer-review process, 13 papers have been accepted to represent the recent advancement of design theories, methodologies, and case studies related to MDO. These papers can be roughly classified into two catalogues: solving strategies and applications.

R. A. Borges et al. discussed the formulation and solution of a system optimization using heuristic methods. Three bioinspired algorithms (Bees Colony algorithm, Firefly Colony algorithm, and Fish Swarm algorithm) are applied and compared to maximize the suppression bandwidth of a nonlinear damped system. MDO involves multiple design criteria, and two alternative ways to deal with the confliction of objectives in linear programming are Pareto set and Data Envelopment Analysis (DEA). M. Li provided a new representation of the Pareto set, which is generic to optimization problems with multi-design criteria. This representation was applied in Data Envelopment Analysis (DEA) models which simplified the derivation and acquisition of the properties associated with the Pareto set. J. W. Wang et al. presented a new algorithm with an integration of the Particle Swarm Optimization and predatory search strategy. The new algorithm was compared with others and showed its advantages in tackling the balance of exploitation and exploration in optimization. The heuristic algorithms can generally deal with any optimization problems; however, they have the limitations of inconsistency and low convergence rate; in particular, it is challenging to be applied in an optimization with expensive fitness functions. A surrogate model can be 
integrated to alleviate these limitations. T. Kunakote and S. Bureerat proposed to integrate the Pareto evolutionary algorithm with several surrogate models. Different integration scenarios were considered for eight structural design problems. Computation and time are the critical measures for the efficiency of optimization methodologies. Time relates to the number of iterations in the solving process. E. Devendorf and $\mathrm{K}$. Lewis discussed the estimation of the number of iterations for distributed design processes; the game theory is integrated with the conventional discrete system theory for approximation.

B. Li et al. developed a methodology to design stiffened plate structures using evolutionary algorithms. X. Liang et al. investigated the effect of shock wave on a plate and associated fluid-structure interaction and thickness effects. An analytical approach was proposed for the transient analysis of a homogenous rectangular plate; the Mindlin plate theory and the Navier solution were integrated with Laplace inversion technique. W. Huang and S. K. Oh proposed the new fuzzy inference systems based on a multiobjective opposition-based space search algorithm (MOSSA); the developed systems are used for system identification to enhance the flexibility of fuzzy models. The MOSSA is a space search algorithm with an opposition-based learning method; this new method employs an "opposite numbers" mechanism to accelerate the convergence in optimization. T. Yifei et al. formulated an energy model of bridge cranes, and the Finite Element Method was used for the structurelevel optimization. Time dependence is an important issue in the design optimization. K. Meruva et al. tackled the formulation of optimization with a consideration of the fatigue life, and solved the design problem with an integration of analytical models and numerical approaches. This work has its significance at both aspects of design and optimization of components and large scale complex system. C. Liu et al. designed a new variable reluctance (VR) resolver for the angle measurement. The models for the structure and output voltages of signal windings are formulated, and Finite Element Analysis (FEA) was applied to predict the machine performance. In optimizing a planetary gear system, Q. Huang et al. proposed a dynamic model, which took into account time-varying mesh stiffness, excitation fluctuation, and gear backlash nonlinearities. The developed nonlinear differential equations were solved by variable step-size Runge-Kutta algorithm, and an optimization model was developed to minimize the system vibration. V. V. Kumar et al. discussed the support of the optimization involved in design of fixtures and product lines; they presented a webbased framework to perform fixture design and assembly line production concurrently. The framework was capable of dealing with the spatial and generational varieties, and computer-assisted tools were embedded to select suppliers and optimize fixtures.

We wish the collected works are helpful to the readers in the field of MDO. Due to the time constraint, we are not able to include more papers covering other important issues such as the formulation of complex optimization systems and the MDO applications in large-scale systems. Readers are encouraged to submit their new contributions to this journal.

\section{Acknowledgments}

The guest editorial team is grateful to the authors of the special issue for their contributions and to the reviewers for their valuable comments on the submissions.

Zhuming Bi

Lihui Wang

Chong $W u$

Guilin Yang

Dan Zhang 


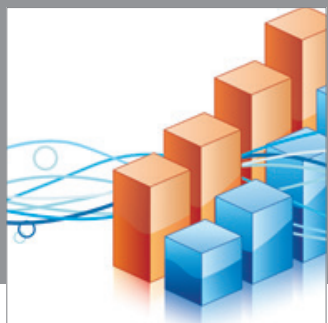

Advances in

Operations Research

mansans

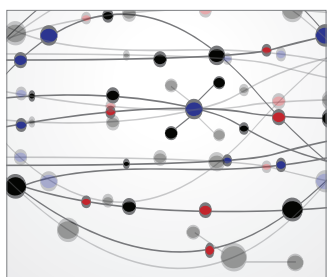

The Scientific World Journal
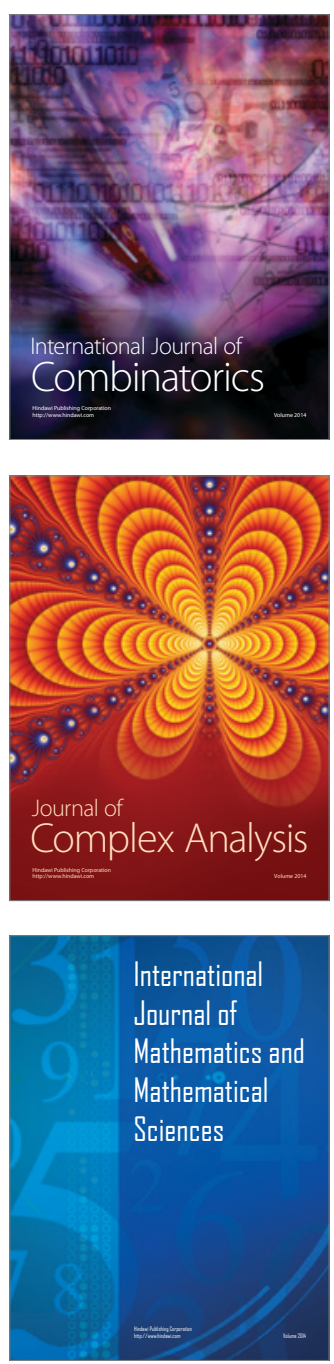
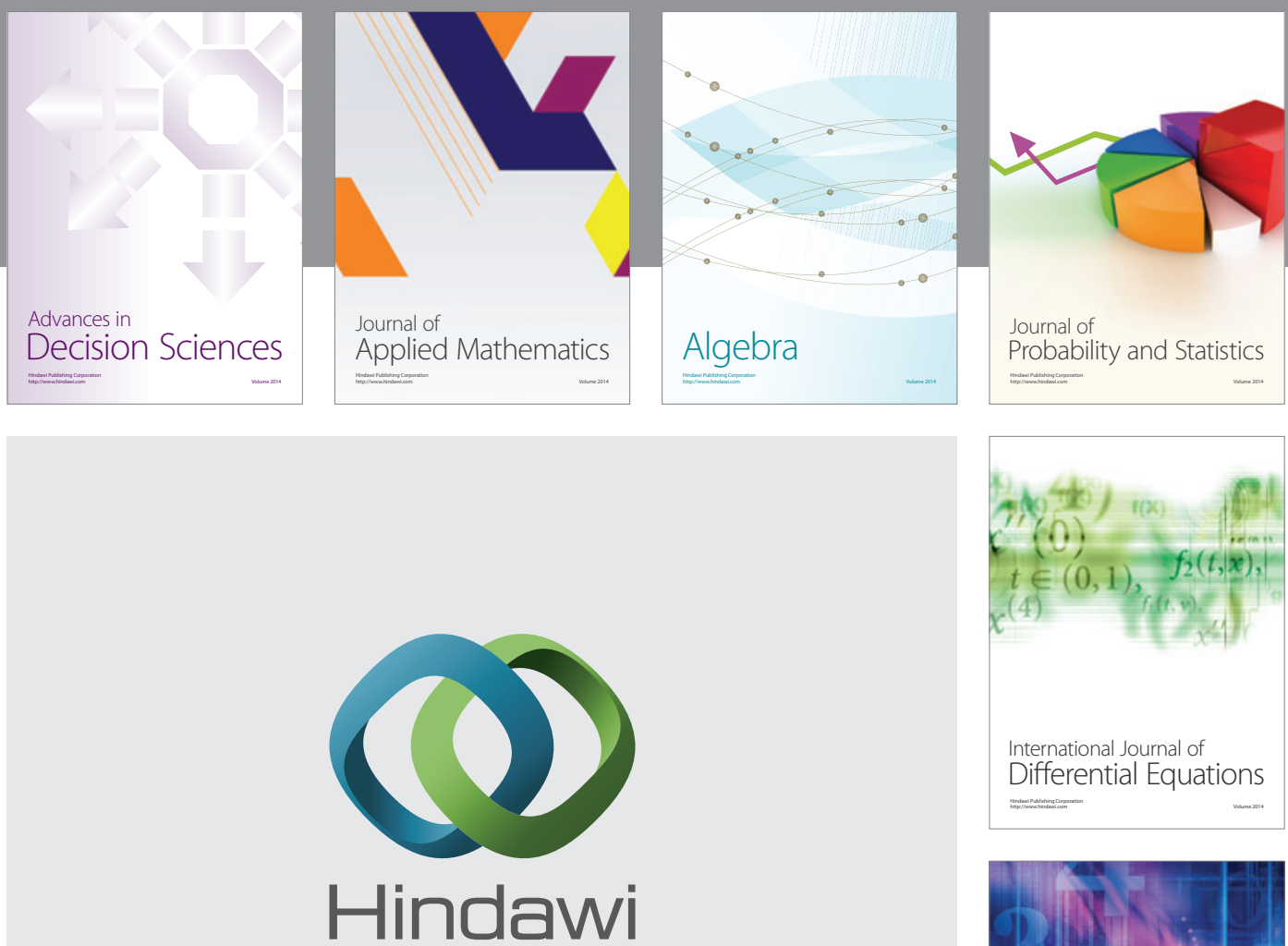

Submit your manuscripts at http://www.hindawi.com
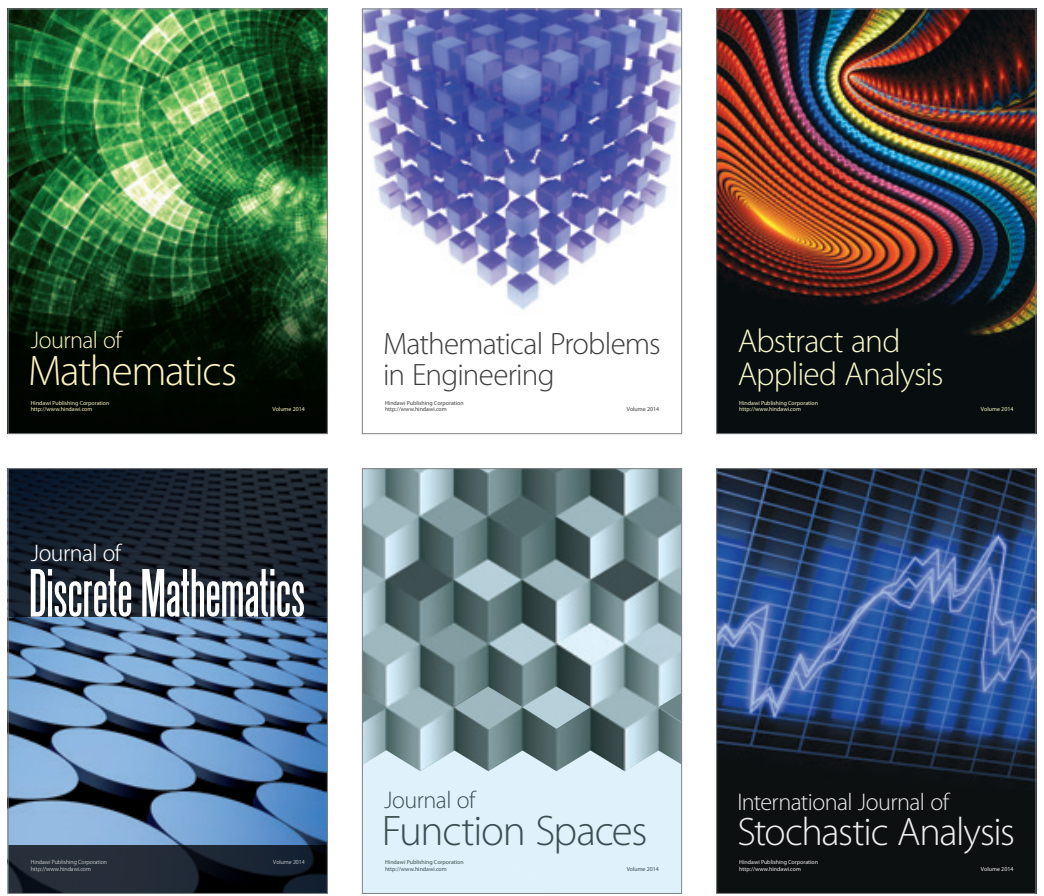

Journal of

Function Spaces

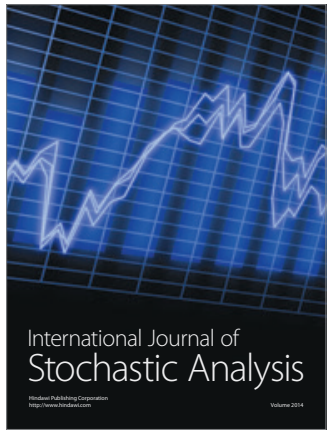

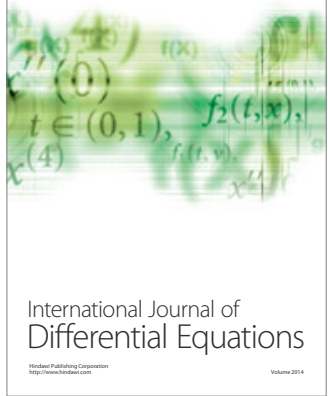
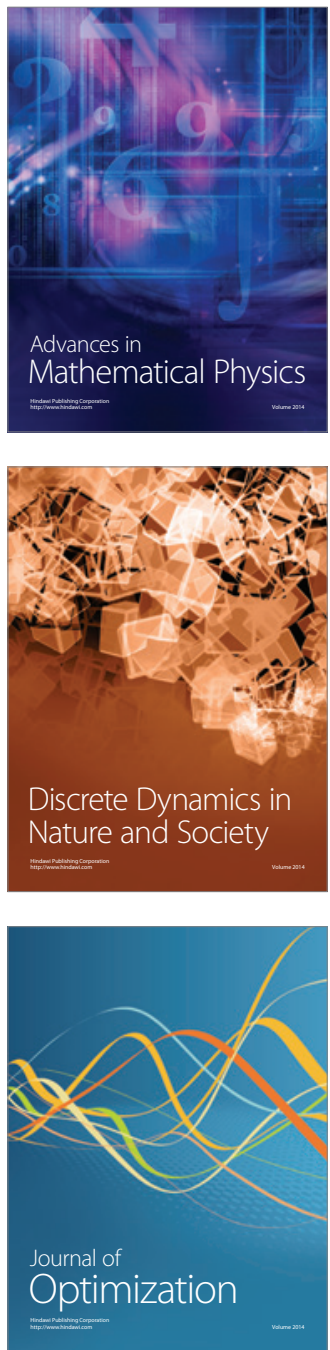